\title{
The effect of zipper-interacting protein kinase on high glucose-stimulated human aortic smooth muscle cells
}

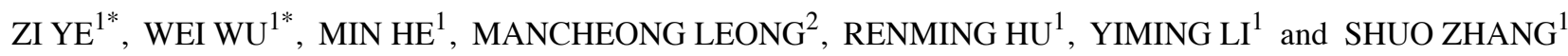 \\ ${ }^{1}$ Institute of Endocrinology and Diabetology, Huashan Hospital, School of Medicine, Fudan University, \\ Shanghai 200040; ${ }^{2}$ Department of Endocrinology, City University of Macau, Macau 000853, P.R. China
}

Received December 19, 2013; Accepted March 4, 2014

DOI: $10.3892 /$ ijmm.2014.1697

\begin{abstract}
Biologic abnormalities in vascular smooth muscle cells (VSMC) may perform a crucial role in the pathogenesis of diabetic vascular disease. The principal aim of this study was to determine the effects of zipper-interacting protein kinase (ZIPK) on human aortic smooth muscle cells (HASMCs) stimulated by high glucose (HG). To elucidate the role of ZIPK in HG-treated HASMCs, we overexpressed ZIPK by lentivirus infection and knocked down ZIPK by gene deletion using ZIPK shRNA. Flow cytometry and Cell Counting kit-8 (CCK-8) were separately used to analyze cell apoptosis and proliferation. Migratory activity was examined using transwell migration chamber assays. The results showed that ZIPK overexpression inhibited cell growth and migration, enhanced cell apoptosis, and reversed cell cycle disturbance by regulating the related proteins of cellular physiological process, such as human cell division cycle 14A phosphatase (Hcdc14A) and intercellular adhesion molecule 1 (ICAM-1). In conclusion, the results suggested that ZIPK plays a role in HG-treated HASMCs, indicating ZIPK is a potential therapeutic target for the treatment of diabetic vascular complications.
\end{abstract}

\section{Introduction}

It has been well documented that diabetic patients have an increased risk of atherosclerotic vascular disease. High glucose (HG) may trigger many cellular events, such as oxidative injury, enhanced mitogenicity, and vascular cell proliferation, which are key processes in the development of atherosclerosis in diabetic vascular complication (1). Diabetic

Correspondence to: Dr Yiming Li or Dr Shuo Zhang, Institute of Endocrinology and Diabetology, Huashan Hospital, School of Medicine, Fudan University, No. 12 Middle Wulumuqi Road, Jingan District, Shanghai 200040, P.R. China

E-mail: yimingli@fudan.edu.cn

E-mail: colleen0708@hotmail.com

*Contributed equally

Key words: zipper-interacting protein kinase, high glucose, human aortic smooth muscle cells, proliferation vascular complications are characterized by the progressive atherosclerotic lesion, and vasculature alteration. The proliferation of vascular smooth muscle cells (VSMC) in the blood vessel wall is a key event in diabetic vascular complications and may play a crucial role in the regulation of blood flow and pressure (2). Furthermore, differentiation and migration of VSMC contribute to development of atherosclerosis in native vessels as well as restenosis formation after vascular injury (3-5).

Zipper-interacting protein kinase (ZIPK), a member of the death-associated protein kinases (DAPKs) family, is isolated from bladder smooth muscle as a $32-\mathrm{kDa}$ phosphoprotein $(6,7)$. Similar to other DAPK family proteins, ZIPK is mainly involved in the regulation of cell death including apoptosis and autophagy (8-10), as well as mitotic processes (11). In addition, several other functions of ZIPK such as the regulation of smooth muscle contraction have been identified. In smooth muscle, ZIPK was found to phosphorylate light chain (LC20) at Ser19 and Thr18 in a $\mathrm{Ca}^{2+} /$ calmodulin-independent manner $(12,13)$. In addition, ZIPK enhances STAT3 transcriptional activity (14), while STAT3 plays a role in cell growth and apoptosis. However, a physiological role for a ZIPK in HG-treated human aortic smooth muscle cells (HASMCs) remains unclear.

The aim of this study, therefore, was to evaluate the proliferation, apoptosis, migration as well as the cell cycle progression of HG-treated HASMCs by overexpressing and knocking down ZIPK, and explore the potential mechanisms involved.

\section{Materials and methods}

Cell culture andmaterials.HASMCs were cultured in Dulbecco's modified Eagle's medium (DMEM) (Gibco, Grand Island, NY, USA) supplemented with $10 \%$ fetal bovine serum (FBS) and $5 \% \mathrm{CO}_{2}$ at $37^{\circ} \mathrm{C}$. Cells (293TN) were cultivated in DMEM (Gibco) with $10 \%$ of FBS. Human lung fibroblast MRC-5 was maintained as a monolayer culture in nutrient medium and Roswell Park Memorial Institute-1640 medium (RPMI-1640) (Sigma-Aldrich, St. Louis, MO, USA). Cell culture reagents, TRIzol reagent, Platinum SYBR-Green qPCR SuperMix -UDG plus ROX and Lipofectamine 2000 were purchased from Invitrogen Life Technologies (Carlsbad, CA, USA). The real-time PCR Master mix kit and the ReverTra Ace kit were purchased from Toyobo (Osaka, Japan). Anti-intercellular adhesion molecule (ICAM)-1, anti-vascular cell adhesion molecule (VCAM)-1, and anti-matrix metalloproteinase (MMP)-2, 
anti-angio-associated migratory cell protein (AAMP) and anti-human cell division cycle gene (Hcdc) 14A antibody were purchased from Abcam (Cambridge, MA, USA).

Determination of apoptosis by flow cytometry. To detect externalized phosphatidylserine (PS) as an early indication of apoptosis, HASMCs $\left(1 \times 10^{5} / \mathrm{ml}\right)$ were incubated for $24 \mathrm{~h}$ at $37^{\circ} \mathrm{C}$. The cells were then collected, washed twice with FACS buffer (0.2\% BSA/PBS), stained with Annexin V-FITC and propidium iodide (PI) according to the manufacturer's instructions, and then analyzed with a Becton-Dickinson (BD) (Franklin Lakes, NJ, USA) FACSCalibur flow cytometer and CellQuest Pro software.

Determination of perturbations of cell cycle. For the cell cycle analysis, the cells were grown in 6-well plates and transfected with plasmids. At $72 \mathrm{~h}$ after transfection, the cells were collected, washed with ice-cold phosphate-buffered saline (PBS), and fixed with 70\% ethanol for at least $1 \mathrm{~h}$. The fixed cells were washed and stained with PI for $1 \mathrm{~h}$ at room temperature. PI-stained nuclei were then analyzed using the BD FACSCalibur system.

Migration assays. Matrigel-coated filter inserts $(8 \mu \mathrm{m}$ pore size) that fit into 24-well migration chambers were obtained from Becton-Dickinson. HASMCs were then plated on the upper chamber. The lower chamber was filled with culture media. Cells in the chamber were incubated for $24 \mathrm{~h}$ at $37^{\circ} \mathrm{C}$ and cells that invaded the lower membrane surface were fixed with methanol and stained with hematoxylin and eosin. The cells that passed through the Matrigel and were located on the underside of the filter were counted. Random fields were counted by light microscopy under a high-power field (x400).

Cell proliferation assay. HASMCs were seeded at a density of $2 \times 10^{4}$ cells/well in 96-well culture plates and cultured in serum-free DMEM supplemented with $10 \%$ fetal bovine serum (FBS), $100 \mathrm{U} / \mathrm{ml}$ penicillin, $100 \mathrm{~g} / \mathrm{ml}$ streptomycin, $8 \mathrm{mM}$ HEPES and $2 \mathrm{mM} \mathrm{L}$-glutamine at $37^{\circ} \mathrm{C}$ in an incubator with a humidified atmosphere of $95 \%$ air and $5 \% \mathrm{CO}_{2}$. Proliferation of the cultured cells was assayed using the Cell Counting kit-8 (CCK-8) (Dojindo Molecular Technologies, Inc., Gaithersburg, MD, USA) according to the manufacturer's instructions $(15,16)$. The rationale for the Cell Counting assay using this specific kit is that the color-developing substrate, WST-8, contained in CCK-8, is reduced by intracellular dehydrogenase to water-soluble formazan, the amount of which can be directly measured photometrically at $450 \mathrm{~nm}$. The cell count has been shown to be linearly proportional to the amount of formazan generated.

Plasmid construction and lentivirus production. Total RNA was extracted from MRC-5 cells using TRIzol and cDNA was prepared by reverse transcription. The ZIPK gene was amplified by PCR with the cDNA. The sequences of the primers used were: 5'-GCTCTAGAGCCACCATGTCCACGTTCAGGCAG-3' and 5'-CGGGATCCCTAGCGCAGCCCGCACTCC-3'. The PCR products were digested by EcoRI and BamHI and a 1589 bp fragment containing ZIPK gene was cloned into the lentiviral vector pCDH-green fluorescent protein (GFP) Lentivector
(System Biosciences, cat. no. CD511A-1). The sequences of the resulting vector (PLV-ZIPK) were verified by sequence analysis.

ZIPK was knocked down with an RNAi technique. The sequence targeting human ZIPK mRNA was chosen based on the full-length mRNA sequence in Genbank (accession no. NM_001348.1). The target sequences of ZIPK (5'-GCCTGG AACATTCCTGGAT-3') were homologous to 806-835 nt of ZIPK mRNA, respectively. The corresponding oligonucleotide templates of the shRNA were chemically synthesized and cloned into the pSIH1-H1-copGFP shRNA vector (System Biosciences, Mountain View, CA, USA) which was digested by BamHI and EcoRI and purified by agarose gel electrophoresis. An invalid RNAi sequence (5'-GAAGCCAGATCCAGC TTCC-3') was used as the negative control. The correct vectors were constructed (designated as pLV-ZIPK and pLV-NC) and confirmed by sequencing.

Virus packaging was performed in 293TN cells after the co-transfection of PLV-ZIPK or PLV-shRNA or PLV-GFP control vector $(500 \mathrm{ng} / \mu \mathrm{l}, 4 \mu \mathrm{l})$ and Lentivirus Package plasmids mix $(500 \mathrm{ng} / \mu \mathrm{l}, 20 \mu \mathrm{l})$ using Lipofectamine 2000. Viruses were harvested $72 \mathrm{~h}$ after transfection, and the highly expressed lentivirus particles were visualized by using green fluorescence. Five types of packaged lentivirus termed by their contents were identified, including pcDNA-ZIPK (ZIPK overexpression), pcDNA (control of pcDNA-ZIPK), shRNA-ZIPK (ZIPK silencing), shRNA-scramzble (scrambled of oligonucleotides as shRNA-ZIPK control) and shRNA (control of shRNA-ZIPK).

Statistical analysis. Experiments were repeated at least three times. The results were expressed as the mean $\pm \mathrm{SD}$ and the data were analyzed using one-way ANOVA followed by a Dunnett's test or Student's t-test to determine any significant differences. $\mathrm{P}<0.05$ was considered to indicate statistical significance.

\section{Results}

Transfection efficiency of recombinant lentivirus in HASMCs. To elucidate the role of ZIPK on HASMCs treated with HG, we overexpressed ZIPK by lentiviral infection and then knocked down ZIPK by gene deletion using ZIPK shRNA. Western blotting was performed to determine the expression of ZIPK. As shown in Fig. 1, the transfection of lentivirus containing pcDNA-ZIPK in HASMCs upregulated ZIPK expression by $\sim 2$-fold, whereas transfection with lentivirus containing shRNA-ZIPK significantly downregulated ZIPK expression.

ZIPK induces apoptosis in HASMCs cultured with HG. The percentage of apoptotic cells in HASMCs was determined by Annexin V/PI staining with flow cytometric analysis. We first investigated the apoptosis of HASMCs in response to treatment with glucose at final concentrations of $25 \mathrm{mmol} / \mathrm{l}$. As shown in Fig. 2, ZIPK overexpression significantly induced apoptosis in HG-treated HASMCs, whereas non-infected control cells and cells infected with shRNA-ZIPK or nonsense shRNA had no effect on the apoptosis of HASMCs.

ZIPK inhibits the proliferation of HASMCs cultured with HG. To determine the effect of ZIPK on HASMC proliferation, we 

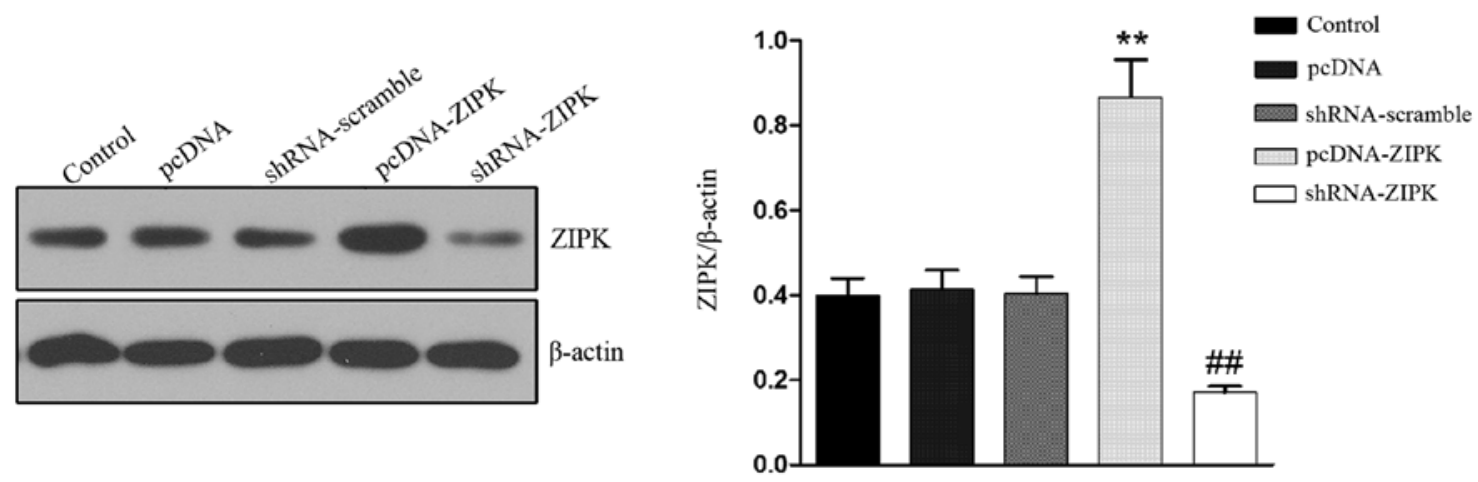

Figure 1. Overexpression and knockdown of zipper-interacting protein kinase (ZIPK) by lentivirus in human aortic smooth muscle cells (HASMCs). HASMCs were infected by lentivirus carrying the ZIPK gene (pcDNA-ZIPK) or shRNA direct against ZIPK (shRNA-ZIPK) or the vehicles. The level of ZIPK protein was determined at 48 -h post-infection by western blotting. The total amounts of these proteins were used for normalization and immunoblots were analyzed by densitometry; the values are given as a percentage of the control. Data were quantified with densitometry and are expressed as the mean $\pm \mathrm{SD}(\mathrm{n}=3){ }^{* * *} \mathrm{P}<0.01$ and ${ }^{\# \#} \mathrm{P}<0.01$ compared with the control group.

A
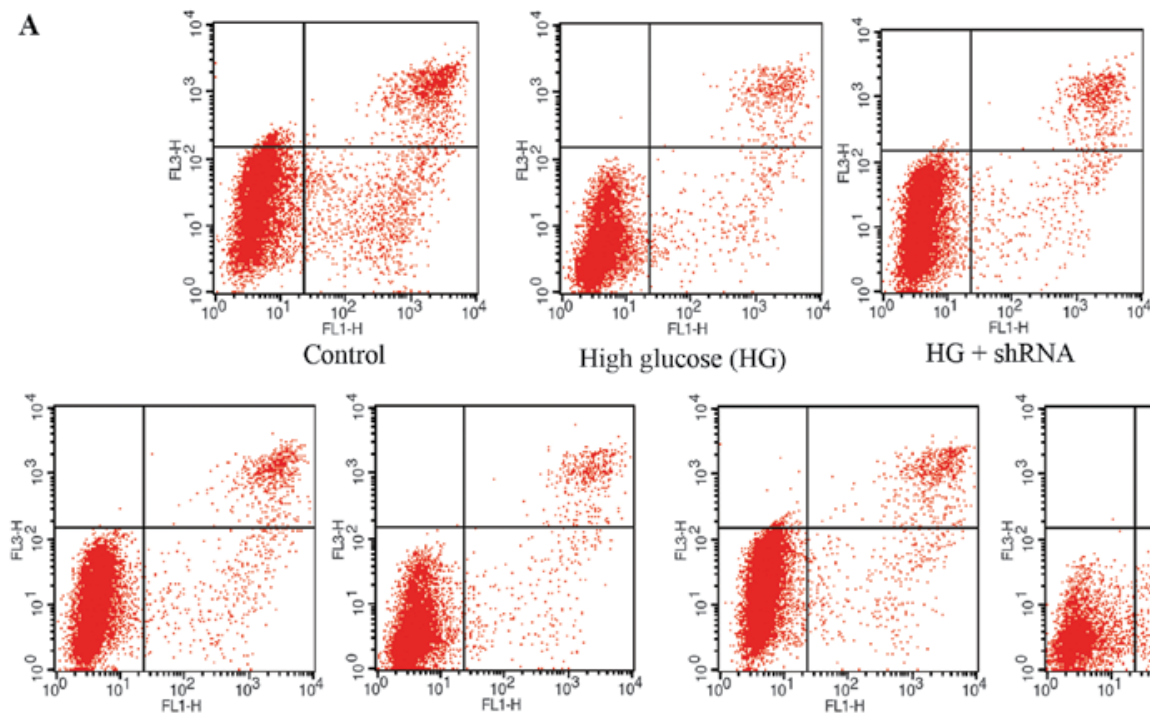

$\mathrm{HG}+$ shRNA-scramble

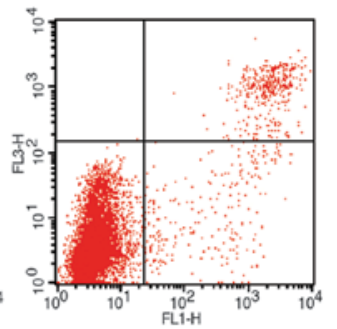

$\mathrm{HG}+$ shRNA-ZIPK
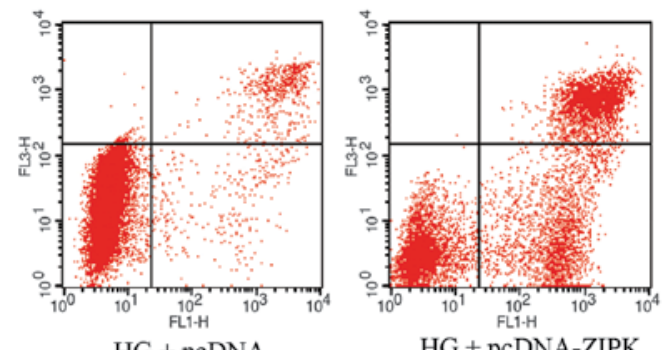

$\mathrm{HG}+\mathrm{pcDNA}$
$\mathrm{HG}+\mathrm{pcDNA}-\mathrm{ZIPK}$

B

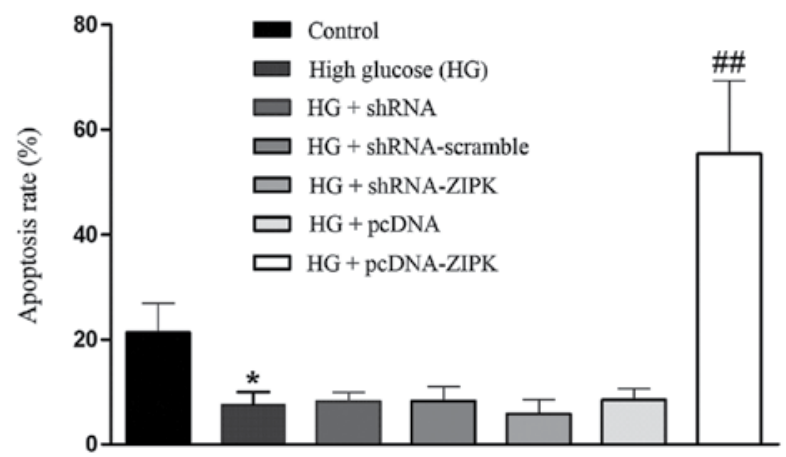

Figure 2. Determination of apoptosis by Annexin V assay. For the apoptosis analysis, human aortic smooth muscle cells (HASMCs) (1x10 $/ \mathrm{ml})$ were stained with Annexin V/propidium iodide (PI), and the apoptotic cell population was evaluated by flow cytometry. (A) Results from one representative experiment are shown. Annexin V staining was presented on the $\mathrm{x}$-axis and PI incorporation was presented on the $\mathrm{y}$-axis. Annexin $\mathrm{V}+\mathrm{PI}^{-}$cells were considered as early apoptotic, while Annexin $\mathrm{V}+\mathrm{PI}^{+}$cells as late apoptotic. The results were expressed as a percentage of the total cells. (B) Results are the mean $\pm \mathrm{SD}$ of three independent experiments [ $\mathrm{P}<0.05$ compared with the control group; ${ }^{\# \#} \mathrm{P}<0.01$ compared with the high glucose (HG) group].

evaluated cell proliferation using the CCK-8. As shown in Fig. 3, exposure of HASMCs to HG stimulated cell proliferation, while shRNA-ZIPK transfection further increased the pro-proliferative effect at 24- and 48-h post-transfection. By contrast, an increase in the proliferation rate stimulated by HG was markedly reduced as a result of pre-treatment with pcDNA-ZIPK at 48 -h post-transfection. The results indicated the protective role of ZIPK in the proliferation of HASMCs stimulated by HG. 


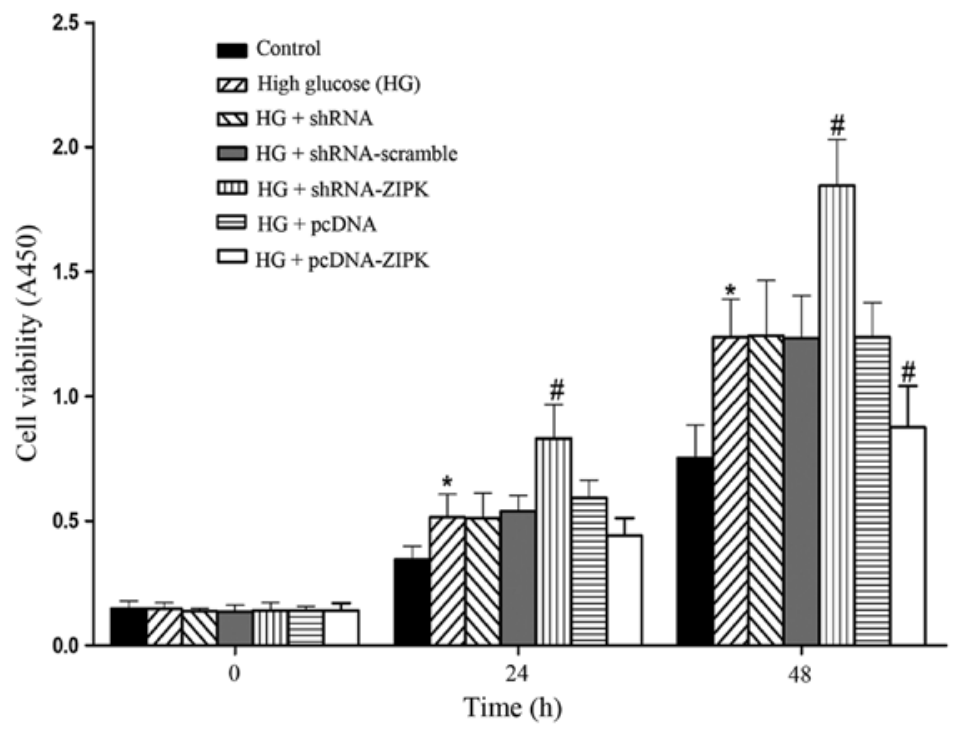

Figure 3. Modulations of the proliferation of human aortic smooth muscle cells (HASMCs) by zipper-interacting protein kinase (ZIPK). HASMC viability at 24- and 48-h post-transfection were measured using the Cell Counting kit-8 according to the manufacturer's instructions. ZIPK treatment inhibited cell proliferation, whereas silencing of ZIPK expression increased cells proliferation. Bar graphs are the mean \pm SD in at least three independent experiments ${ }^{*} \mathrm{P}<0.05$ compared with the control group; ${ }^{~} \mathrm{P}<0.05$ compared with the high glucose (HG) group.

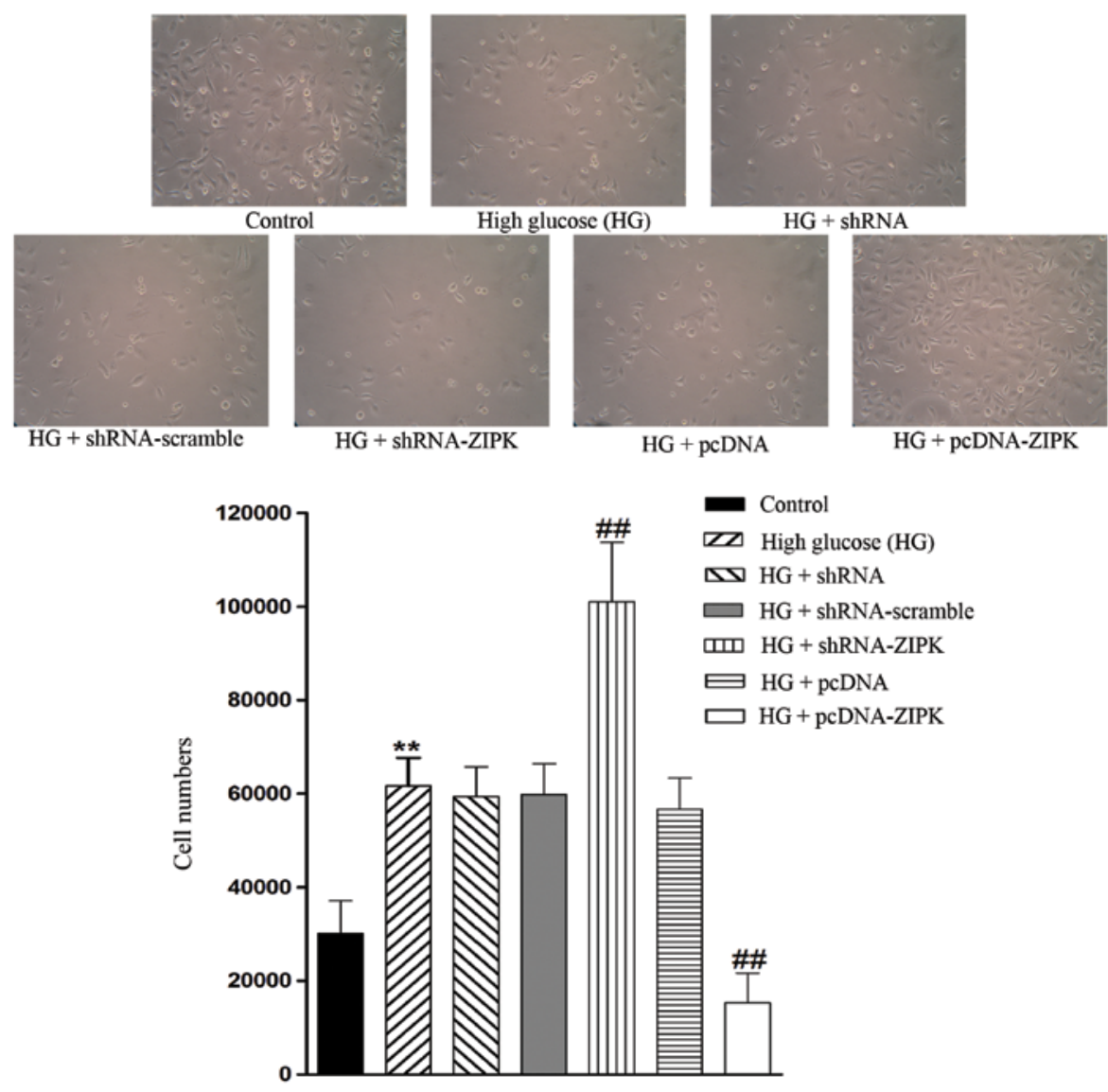

Figure 4. Effect of zipper-interacting protein kinase (ZIPK) on the migration of human aortic smooth muscle cells (HASMCs). Three independent experiments were performed with six chambers in each time period and the number of cells in eight standardized fields was counted in each chamber. Changes in cell morphology were observed microscopically; original magnification, x200. ZIPK overexpression resulted in a marked decreased migratory activity, whereas silencing of ZIPK expression showed significantly increased migratory activity. ${ }^{* *} \mathrm{P}<0.01$ compared with the control group; ${ }^{\# \prime} \mathrm{P}<0.01$ compared with the high glucose (HG) group.

ZIPK decreases the migration ability of HG-treated HASMCs. To delineate the role of ZIPK in migration, we separately used lentivirus as a vector to downregulate and upregulate ZIPK expression. As shown in Fig. 4, the migration of HASMCs was promoted by treatment with HG as compared with the control, and infection with shRNA-ZIPK further enhanced 


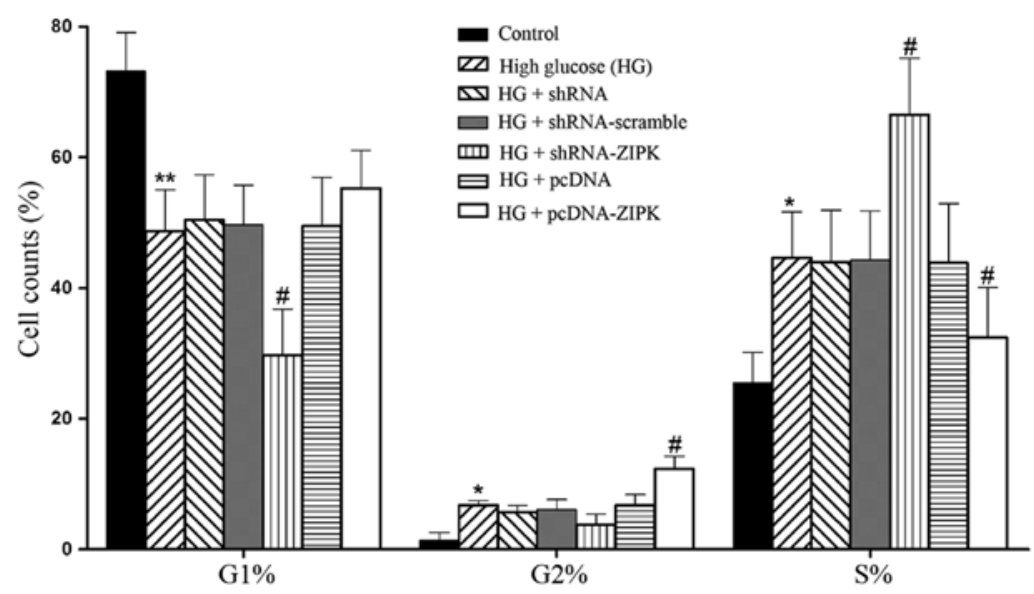

Figure 5. Effect of zipper-interacting protein kinase (ZIPK) on cell cycle progression in human aortic smooth muscle cell (HASMC). HASMC were collected following $24 \mathrm{~h}$ treatment with lentivirus containing ZIPK or ZIPK inhibitor or the control. Bar graphs are the mean \pm SD in at least three independent experiments. ${ }^{*} \mathrm{P}<0.05$ and ${ }^{* *} \mathrm{P}<0.01$ compared with the control group; ${ }^{*} \mathrm{P}<0.05$ compared with the high glucose (HG) group, calculated by the Student's t-test, indicating statistically significant differences.
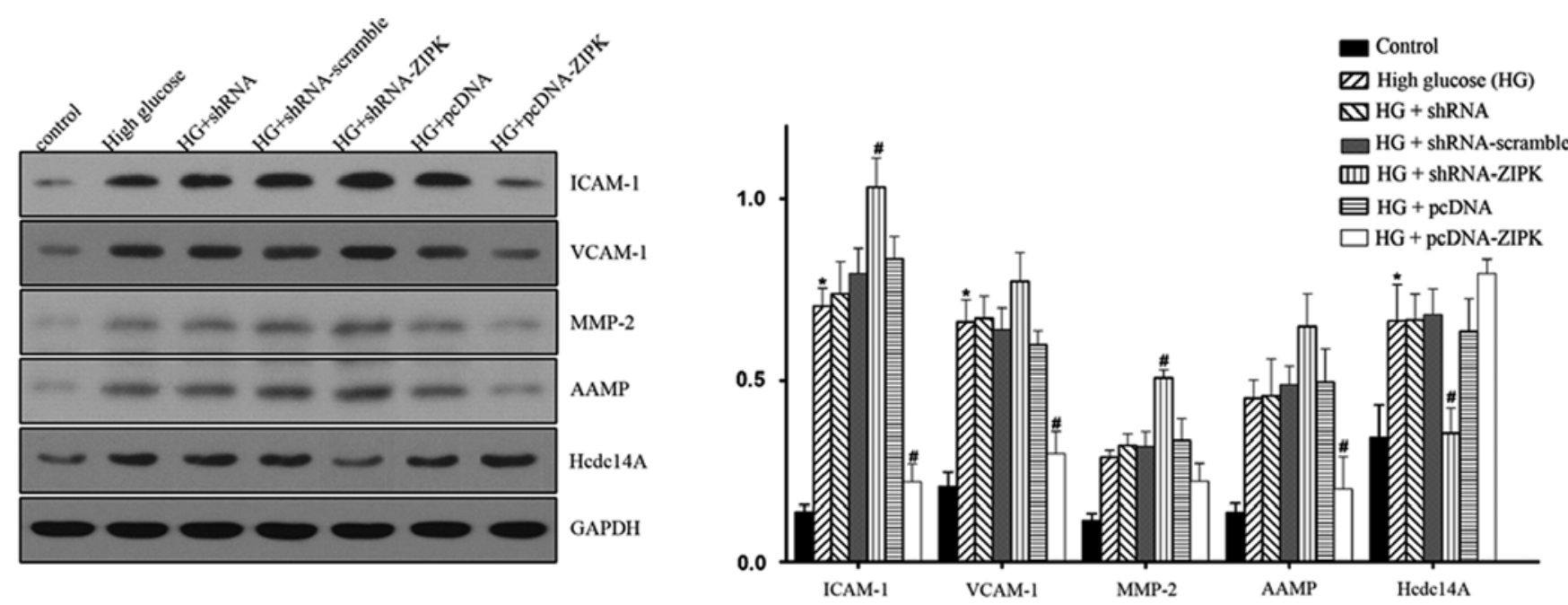

Figure 6. Modulations of cell cycle, apoptosis and migration-related proteins by zipper-interacting protein kinase (ZIPK) in high glucose (HG)-treated human aortic smooth muscle cells (HASMCs). HASMCs were transfected with ZIPK or ZIPK inhibitor or the control lentivirus. Western blotting was performed with specific antibodies. The data are expressed as the means $\pm \mathrm{SD}$ of three independent experiments. ${ }^{*} \mathrm{P}<0.01$ vs. control, ${ }^{\#} \mathrm{P}<0.05$ vs. HG alone.

the migration rate, whereas ZIPK overexpression markedly inhibited HG-treated HASMC invasion. These experiments indicated that ZIPK may perform an inhibitory role in the process of HASMC invasion.

ZIPK participates in the mitotic progression of HASMCs. Since ZIPK has been associated with the regulation of mitotic processes (11), we assessed the function of ZIPK in HASMCs on cell cycle with the HG stimulation. Results are presented as diagrams of cell distribution over the cell cycle phases of HASMCs. As shown in Fig. 5, HG treatment induced arrest in the $\mathrm{S}$ phase and decreased the cell percentage in the $\mathrm{G} 1$ and G2 phase of the cell cycle as compared with the non-treated control. Silencing of ZIPK expression markedly induced arrest in the $\mathrm{S}$ phase, whereas HASMCs treated with pcDNA-ZIPK attenuated S-phase retardation and retarded the cell cycle progression through an increased G2 phase compared to the HG group. These data suggested that the overexpression of
ZIPK exerted a protective effect on cell cycle disturbance of HASMCs stimulated by HG.

Modulations of cell cycle, apoptosis and migration-related proteins by ZIPK in HG-treated HASMCs. To explain the potential mechanisms of ZIPK in HASMCs, we examined the expression of several pivotal mediators of the signaling pathway on cell apoptosis, migration, proliferation and cell cycle, including ICAM-1, VCAM-1, MMP-2, AAMP and Hcdc14A by western blotting. The results in Fig. 6 show that stimulation with HG increased the levels of ICAM-1, VCAM-1 and Hcdc14A expression. Infection of shRNA-ZIPK led to the upregulation of ICAM-1, VCAM-1, MMP-2 and AAMP, while the expression of Hcdc14A was downregulated. By contrast, ZIPK pre-treatment resulted in the downregulated expression of ICAM-1, VCAM-1, MMP-2 and AAMP, and the upregulated expression of Hcdc14A. The present investigation demonstrated that gene transfer of ZIPK into HASMCs is a 
regulatory element in the cell physiological process. However, the direct effect of ZIPK remains to be determined.

\section{Discussion}

Patients with diabetes are at higher risk of atherosclerotic disease than non-diabetic individuals with other comparable risk factors. The proliferation and migration of HASMCs play important roles in atherosclerosis during the processes inherent to vascular disease $(17,18)$. To the best of our knowledge, this is the first study to focus on the effect of the overexpression and knocking down of ZIPK by lentivirus in diverse cell processes, including apoptosis, proliferation, migration and the cell cycle in HASMCs treated with HG. ZIPK overexpression led to decreased migration and cell growth, increased apoptosis and ameliorated cell cycle disturbance in HG-treated HASMCs. Our results raise the possibility that ZIPK is a novel potential therapeutic target for the treatment of diabetic microvascular complication.

Findings of previous studies showed that full-length ZIPK may induce diphosphorylation of LC20 and contribute to cell motility and apoptosis $(12,13)$. In addition, ZIPK plays a pivotal role in the regulation of actin filament reorganization, and control of $\mathrm{Ca}^{2+}$ sensitization to induce cell smooth muscle contraction $(13,19)$. Recent accumulating evidence suggests that ZIPK plays a variety of roles in cell death as well as cardiovascular pathophysiology. Cho et al demonstrated in mesenteric arteries of spontaneously hypertensive rats (SHR) that ZIPK modulated calyculin A-induced contraction by increasing $\mathrm{Ca}^{2+}$-independent myosin LC (MLC) kinase activity (20). Moreover, ZIPK has been reported to promote ROS-dependent vascular inflammation and mediate the development of hypertension in SHR (21).

To gain a better understanding of whether ZIPK contributed to HASMC proliferation, apoptosis, migration as well as cell cycle in an HG culture medium, we used two different approaches: shRNA-ZIPK to downregulate and pcDNA-ZIPK to upregulate ZIPK expression. The results showed that ZIPK overexpression decreased HASMC proliferation and the migration rate. Moreover, lentivirus-mediated ZIPK significantly induced HASMC apoptosis treated by HG. The cell cycle experiments showed that inhibition of the expression of ZIPK incurred arrest at the $\mathrm{S}$ phase, thereby accelerating cell proliferation, while ZIPK overexpression reversed the phenomenon of disturbing the cell cycle due to HG. Therefore, the current data emphasized the possibility that recombinant lentivirus-mediated ZIPK gene expression is an effective method of inhibiting HASMC growth, restraining cell migration, inducing apoptosis as well as ameliorating cell cycle disorder in vitro and identified ZIPK as an important component in cellular physiological processes.

Increased proteolytic activity in the vessel wall mediates the degradation of the extracellular matrix surrounding the VSMC in response to HG injury. To clarify the potential mechanisms of ZIPK in the proliferation, apoptosis, migration and cell progression of HASMCs stimulated by HG, we examined several key regulatory molecules: VCAM1 and ICAM-1 $(22,23)$, together with AAMP proteins $(24)$, have been strongly suggested to play an important role in cell migration; Hcdc14A is shown to interact with interphase centrosomes and to regulate the centrosome duplication cycle $(25,26)$; MMP-2 caused cell proliferation (27), while the activation of MMP, particularly the gelatinases MMP-2, may contribute to the pathogenesis of atherosclerosis by facilitating the migration of VSMC $(28,29)$. Our data have shown that ZIPK overexpression resulted in the a decreased expression of ICAM-1, VCAM-1, MMP-2 and AAMP, and an elevated expression of Hcdc14A. Potential explanations for this decrease and increase in expression may be : i) ZIPK may inhibit cell migration by the regulation of multiple downstream signaling components including ICAM-1, VCAM-1, AAMP and MMP-2; ii) ZIPK participated in cell cycle progression by interacting with Hcdc14A and iii) ZIPK inhibited HASMC proliferation presumably by the downregulation of MMP-2. However, further mechanistic explorations to elucidate the signaling mechanisms of ZIPK in HASMCs are required.

Of note, ZIPK overexpression upregulated the level of Hcdc14A, which was consistent with results of a previous study demonstrating that ZIPK physically interacted with Hcdc14A and ZIPK-mediated phosphorylation was able to initiate the phosphatase activity of Hcdc14A (data not shown). Hcdc14A phosphatase plays a role in the regulation of the centrosome cycle, mitosis, and cytokinesis and overproduction of Hcdc14A caused mitotic spindle and chromosome segregation (30). This finding may explain the reason for the overexpression of ZIPK attenuating disturbance of the cell cycle.

In conclusion, our results demonstrate the protective function of ZIPK in HASMCs under HG conditions and its potential mechanisms. The results provide insight to a novel therapeutic strategy for diabetic vascular complications.

\section{Acknowledgements}

This study was supported by the National Nature Science Foundation for Young Scientists of China (no. 30900541) and 985 engineering advantage subject innovation platform (no. 985III).

\section{References}

1. Ruderman NB and Haudenschild C: Diabetes as an atherogenic factor. Prog Cardiovasc Dis 26: 373-412, 1984.

2. Srivastava AK: High glucose-induced activation of protein kinase signaling pathways in vascular smooth muscle cells: a potential role in the pathogenesis of vascular dysfunction in diabetes (review). Int J Mol Med 9: 85-89, 2002.

3. Blindt R, Krott N, Hanrath P, vom Dahl J, van Eys G and Bosserhoff AK: Expression patterns of integrins on quiescent and invasive smooth muscle cells and impact on cell locomotion. J Mol Cell Cardiol 34: 1633-1644, 2002.

4. Ross R: Cell biology of atherosclerosis. Annu Rev Physiol 57: 791-804, 1995.

5. Owens GK, Kumar MS and Wamhoff BR: Molecular regulation of vascular smooth muscle cell differentiation in development and disease. Physiol Rev 84: 767-801, 2004.

6. Kawai T, Matsumoto M, Takeda K, Sanjo H and Akira S: ZIP kinase, a novel serine/threonine kinase which mediates apoptosis. Mol Cell Biol 18: 1642-1651, 1998.

7. Kögel D, Plöttner O, Landsberg G, Christian S and Scheidtmann KH: Cloning and characterization of Dlk, a novel serine/threonine kinase that is tightly associated with chromatin and phosphorylates core histones. Oncogene 17: 2645-2654, 1998.

8. Brognard J,Zhang YW, Puto LA and Hunter T: Cancer-associated loss-of-function mutations implicate DAPK3 as a tumorsuppressing kinase. Cancer Res 71: 3152-3161, 2011. 
9. Mills JC, Stone NL, Erhardt J and Pittman RN: Apoptotic membrane blebbing is regulated by myosin light chain phosphorylation. J Cell Biol 140: 627-636, 1998.

10. Coleman ML, Sahai EA, Yeo M, Bosch M, Dewar A and Olson MF: Membrane blebbing during apoptosis results from caspase-mediated activation of ROCK I. Nat Cell Biol 3 : 339-345, 2001.

11. Engemann H, Heinzel V, Page G, Preuss U and Scheidtmann KH: DAP-like kinase interacts with the rat homolog of Schizosaccharomyces pombe CDC5 protein, a factor involved in pre-mRNA splicing and required for $\mathrm{G} 2 / \mathrm{M}$ phase transition. Nucleic Acids Res 30: 1408-1417, 2002.

12. Murata-Hori M, Suizu F, Iwasaki T, Kikuchi A and Hosoya $\mathrm{H}$ : ZIP kinase identified as a novel myosin regulatory light chain kinase in HeLa cells. FEBS Lett 451: 81-84, 1999.

13. Niiro $\mathrm{N}$ and Ikebe $\mathrm{M}$ : Zipper-interacting protein kinase induces $\mathrm{Ca}(2+)$-free smooth muscle contraction via myosin light chain phosphorylation. J Biol Chem 276: 29567-29574, 2001.

14. Sato N, Kawai T, Sugiyama K, Muromoto R, Imoto S, Sekine Y, Ishida M, Akira $S$ and Matsuda T: Physical and functional interactions between STAT3 and ZIP kinase. Int Immunol 17: 1543-1552, 2005.

15. Kakudo N, Shimotsuma A, Miyake S, Kushida S and Kusumoto K : Bone tissue engineering using human adipose-derived stem cells and honeycomb collagen scaffold. J Biomed Mater Res A 84 191-197, 2008

16. Kakudo N, Minakata T, Mitsui T, Kushida S, Notodihardjo FZ and Kusumoto K: Proliferation-promoting effect of platelet-rich plasma on human adipose-derived stem cells and human dermal fibroblasts. Plast Reconstr Surg 122: 1352-1360, 2008.

17. Yasunari K, Kohno M, Kano H, Yokokawa K, Minami M and Yoshikawa $\mathrm{J}$ : Mechanisms of action of troglitazone in the prevention of high glucoseinduced migration and proliferation of cultured coronary smooth muscle cells. Circ Res 81 : 953-962, 1997.

18. Suh SJ, Jin UH, Kim SH, Chang HW, Son JK, Lee SH, Son KH and Kim CH: Ochnaflavone inhibits TNF-alpha-induced human VSMC proliferation via regulation of cell cycle, ERK1/2, and MMP-9. J Cell Biochem 99: 1298-1307, 2006.

19. Komatsu S and Ikebe M: ZIP kinase is responsible for the phosphorylation of myosin II and necessary for cell motility in mammalian fibroblasts. J Cell Biol 165: 243-254, 2004.

20. Cho YE, Ahn DS, Morgan KG and Lee YH: Enhanced contractility and myosin phosphorylation induced by $\mathrm{Ca}(2+)$-independent MLCK activity in hypertensive rats. Cardiovasc Res 91: 162-170, 2011
21. Usui T, Okada M, Hara Y and Yamawaki H: Death-associated protein kinase 3 mediates vascular inflammation and development of hypertension in spontaneously hypertensive rats. Hypertension 60: 1031-1039, 2012.

22. Piconi L, Quagliaro L, Da Ros R, Assaloni R, Giugliano D, Esposito K, Szabó C and Ceriello A: Intermittent high glucose enhances ICAM-1, VCAM-1, E-selectin and interleukin-6 expression in human umbilical endothelial cells in culture: the role of poly(ADP-ribose) polymerase. J Thromb Haemost 2: 1453-1459, 2004.

23. Martinelli R, Gegg M, Longbottom R, Adamson P, Turowski P and Greenwood J: ICAM-1-mediated endothelial nitric oxide synthase activation via calcium and AMP-activated protein kinase is required for transendothelial lymphocyte migration. Mol Biol Cell 20: 995-1005, 2009.

24. Vogt F, Zernecke A, Beckner M, Krott N, Bosserhoff AK, Hoffmann R, Zandvoort MA, Jahnke T, Kelm M, Weber C and Blindt R: Blockade of angio-associated migratory cell protein inhibits smooth muscle cell migration and neointima formation in accelerated atherosclerosis. J Am Coll Cardiol 52: 302-311, 2008

25. Mailand N, Lukas C, Kaiser BK, Jackson PK, Bartek J and Lukas J: Deregulated human Cdc14A phosphatase disrupts centrosome separation and chromosome segregation. Nat Cell Biol 4: 317-322, 2002

26. Kaiser BK, Zimmerman ZA, Charbonneau H and Jackson PK: Disruption of centrosome structure, chromosome segregation, and cytokinesis by misexpression of human Cdc14A phosphatase. Mol Biol Cell 13: 2289-2300, 2002.

27. Galli A, Svegliati-Baroni G, Ceni E, Milani S, Ridolfi F, Salzano R, Tarocchi M, Grappone C, Pellegrini G, Benedetti A, Surrenti $\mathrm{C}$ and Casini A: Oxidative stress stimulates proliferation and invasiveness of hepatic stellate cells via a MMP2-mediated mechanism. Hepatology 41: 1074-1084, 2005.

28. Shah PK: Role of inflammation and metalloproteinases in plaque disruption and thrombosis. Vasc Med 3: 199-206, 1998.

29. Tartakover-Matalon S, Cherepnin N, Kuchuk M, Drucker L, Kenis I, Fishman A, Pomeranz M and Lishner M: Impaired migration of trophoblast cells caused by simvastatin is associated with decreased membrane IGF-I receptor, MMP2 activity and HSP27 expression. Hum Reprod 22: 1161-1167, 2007.

30. Kaiser BK, Zimmerman ZA, Charbonneau H and Jackson PK: Disruption of centrosome structure, chromosome segregation, and cytokinesis by misexpression of human Cdc14A phosphatase. Mol Biol Cell 13: 2289-2300, 2002. 